Research Paper

\title{
Myotilin, a New Topotecan Resistant Protein in Ovarian Cancer Cell Lines
}

\author{
Karolina Sterzyńska1*® ${ }^{1 *}$ Andrzej Klejewski²,3*, Karolina Wojtowicz ${ }^{1}$, Monika Świerczewska1 ${ }^{1}$ Michał \\ Nowicki ${ }^{1}$, Jacek Brązert ${ }^{3}$ and Radosław Januchowski ${ }^{1}$ \\ 1. Department of Histology and Embryology, Poznan University of Medical Sciences, Poznań, Poland \\ 2. Department of Nursing, Poznan University of Medical Sciences, Poznań, Poland \\ 3. Department of Obstetrics and Women's Diseases, Poznan University of Medical Sciences, Poznań, Poland \\ *These authors have contributed equally \\ $\square$ Corresponding author: Karolina Sterzyńska, mailing address: Department of Histology and Embryology, Poznan University of Medical Sciences, \\ Święcickiego 6 St., post code 61-781, Poznań, Poland; email address: k.olejniczak@ump.edu.pl \\ (c) Ivyspring International Publisher. This is an open access article distributed under the terms of the Creative Commons Attribution (CC BY-NC) license \\ (https:// creativecommons.org/licenses/by-nc/4.0/). See http://ivyspring.com/terms for full terms and conditions.
}

Received: 2018.05.18; Accepted: 2018.08.24; Published: 2018.10.22

\begin{abstract}
Background: Low effectiveness of chemotherapy in ovarian cancer results from development of drug resistance during treatment. Topotecan (TOP) is a chemotherapeutic drug used in second-line chemotherapy of this cancer. Unfortunately, during treatment cancer can develop diverse cellular and tissue specific mechanisms of resistance to cytotoxic drugs.

Methods: We analyzed development of TOP resistance in ovarian cancer cell lines (A2780 and WI). On the base of our previous results where a set of "new genes" with different functions that can be related to TOP-resistance was described hereby we performed detailed analysis of MYOT expression. MYOT mRNA level (real time PCR analysis), protein expression in cell lysates and cell culture medium (western blot analysis) and protein expression in cancer cells (immunofluorescence analysis) were determined in this study.

Results: We observed increased expression of MYOT in TOP resistant cell lines at both mRNA and protein level. MYOT, together with extracellular matrix molecules like COL1A2 and COL15A1 were also secreted to corresponding cell culture media.

Conclusion: Our results suggest that upregulation of MYOT can be related to TOP resistance in ovarian cancer cell lines.
\end{abstract}

Key words: myotilin, ovarian cancer, topotecan resistance

\section{Introduction}

Epithelial ovarian cancer (EOC) is the most leathal gynecological malignancy. Poor prognosis results from a late diagnosis, usually at stage III or IV according to FIGO classification and development of resistance to chemotherapy [1]. Although most patients respond well to cytotoxic chemotherapy at the beginning of treatment eventually they develop drug resistance in most cases [1,2]. For most patients the surgery following chemotherapy is the main option of treatment [2]. In the first line chemotherapy regimens combined chemotherapy of platinum and taxanes is used [3]. The second line chemotherapy is based on the patient's response to first line and is composed of platinum, taxanes, doxorubicin, gemcitabine or topotecan (TOP) [4-6]. Unfortunately, most patients with ovarian cancer eventually develop drug resistance leading ineffectiveness of further treatment.

Mechanisms of cancer drug resistance can be divided into two main groups. 1) Mechanism specific to cancer cells like: decreased accumulation of the drug in the cancer cell, change of drug cellular localization, faster inactivation of drug, faster repair of DNA and other cellular components, mutations in 
genes encoding target proteins. However, the most significant mechanism of drug resistance at the cellular level is the expression of drug transporters from the ABC family [7]. Among them the most important are glycoprotein $\mathrm{P}$ (P-gp) and breast cancer resistant protein (BCRP) and both are involved in TOP-resistance [8, 9]. 2) Mechanism specific to cancer tissue. Tumor tissue can be an effective barrier to drug diffusion because of dense cellular structure [10], growth-induced solid stress [11] and expression of extracellular matrix (ECM) components like proteoglycans and collagens [12]. It has been reported that some cytotoxic drugs like Paclitaxel (PAC), Doxorubicin (DOX), Methotrexate (MTX) and Vinblastine (VIN) can bind to cellular molecules making them not available for tumor tissue [12]. Components of ECM not only block drug diffusion but also interact with cancer cells and inhibit their sensitivity to apoptosis [13]. This phenomenon is designated as a cell adhesion-mediated drug resistance (CAM-DR) [14] and was observed in vivo [15] and in vitro [16]. Expression of ECM components has also been reported in drug resistant ovarian [17, 18] and breast [19] cancer cell lines, which indicates that expression of ECM components may play a crucial role in tissue mediated drug resistance as well as drug resistance at cellular level.

Topotecan is an anticancer drug used in the treatment of ovarian cancer [5], cervical cancer [20] and small cell lung carcinoma [21]. It is a semisynthetic derivatives of alkaloid camptothecin, isolated from extracts of the tree Camptotheca acuminate [22]. Topotecan inhibits activity of DNA topoisomerase I, an enzyme involved in DNA helix overwinding or underwinding in the cell nucleus [23]. Stabilization of the enzyme-DNA complex results in blocking of DNA replication and transcription and in consequence leads to cancer cell death [24]. Unfortunately, cancer can develop different mechanisms of TOP-resistance during chemotherapy. Downregulation of topoisomerases I expression or mutation in their genes makes these enzymes less sensitive to drug action [25]. However, the most significant mechanism of TOP-resistance at cellular level is an active transport of this agent from cancer cells. The most important drug transporter playing role in this process is BCRP although we also observed P-gp-dependent TOP-resistance [8, 9]. Among tissue-specific mechanisms of drug resistance the expression of ECM components like lumican [26] or/and different collagens [27] seems to play a considerable role. Recently we also described a set of "new genes" with different functions that can be related to TOP-resistance [28, 29].

Myotilin (MYOT) is encoded by MYOT gene located on chromosome $5 \mathrm{q} 31.2$. This is a $55.3 \mathrm{kDa}$ protein containing of 498 amino acids. It comprises two C2-type Ig domains flanked by a unique serine-rich $\mathrm{N}$-terminus and a short C-terminal tail. This is a structural protein with expression restricted to skeletal and cardiac muscle [30]. It localizes to the sarcomeric $\mathrm{Z}$ discs and interacts with structural proteins like: actinin [31], filamins [32] and FATZ proteins [33] among others. It has been observed that MYOT is mutated in different forms of muscular dystrophy [34]. The best of our knowledge the role of MYOT in drug resistance or even in any cancer type has not been described so far.

Our previous microarray results indicated that MYOT was overexpressed in three TOP-resistant ovarian cancer cell lines [35]. In this study, we performed detailed analysis of MYOT expression at mRNA and protein levels in A2780 (commercially available) and W1 (primary ovarian cancer cell line established in our laboratory) TOP-resistant ovarian cancer cell lines and in their corresponding media. Our results indicate that MYOT can be a novel gene involved in TOP-resistance in ovarian cancer.

\section{Materials and Methods}

\section{Reagents and Antibodies}

TOP was obtained from Sigma (St. Louis, MO, USA). RPMI-1640 and MEM medium, fetal bovine serum, antibiotic-antimycotic solution, and Lglutamine were also purchased from Sigma (St. Louis, MO, USA). Mouse monoclonal anti-MYOT Ab (B-3), goat polyclonal anti-COL1A2 Ab (M-19) and goat polyclonal anti-COL15A1Ab (N-20) were obtained from Santa Cruz Biotechnology (Santa Cruz, CA, US). Donkey anti-goat horseradish peroxidase- (HRP) conjugated $\mathrm{Ab}$ was purchased from Santa Cruz Biotechnology (Santa Cruz, CA, USA). The MFP488 fluorescent secondary antibody was obtained from MoBiTec (Goettingen, Germany). The mounting medium with DAPI was obtained from Santa Cruz Biotechnology (Santa Cruz, CA, US). Columns for protein isolation from serum were purchased from Merck Millipore (Billerica, MA, USA). Western blot reagents (membranes, gels and protein marker) were purchased from Biorad (Bio-Rad Laboratories, Hemel Hempstead, UK).

\section{Cell lines and cell culture}

In our study we used two ovarian cancer cell lines: the established ovarian cancer cell line A2780 and the primary ovarian cancer cell line W1. The human ovarian carcinoma A2780 cell line were purchased from ATCC (American Type Culture Collection, Manassas, VA, USA). A2780 sublines that were resistant to TOP [A2780TR1 and A2780TR2 
(A2780 topotecan resistant)] were generated by exposing A2780 cells to TOP at incrementally increasing concentrations. The human primary ovarian cancer cell line W1 was established in our laboratory using ovarian cancer tissue obtained from an untreated patient. W1 subline resistant to TOP [W1TR (W1 topotecan resistant)] was obtained by exposing W1 cells to TOP at incrementally increasing concentrations. The final concentration used for selecting the resistant cells was $24 \mathrm{ng} / \mathrm{ml}$ of TOP and was two-fold higher than the plasma concentrations of the TOP two hours after intravenous administration. The increase in resistance according to parental drug sensitive cell lines were as follow: 59.6 fold for A2780TR1 vs A2780 and 48.5 fold for A2780T2 vs A2780; 20.0 fold for W1TR vs W1, as described previously $[8,9]$. All of the cell lines were maintained as monolayers in complete medium [MEM medium (A2780), and RPMI-1640 medium (W1) supplemented with $10 \%(\mathrm{v} / \mathrm{v})$ fetal bovine serum, $2 \mathrm{pM}$ L-glutamine, penicillin (100 units/ml), streptomycin (100 units/ml) and amphotericin B $(25 \mu \mathrm{g} / \mathrm{ml})]$ at $37^{\circ} \mathrm{C}$ in a $5 \% \mathrm{CO}_{2}$ atmosphere.

\section{Examination of Gene Expression Using QPCR}

The changes in MYOT expression in the A2780, W1 and drug-resistant cell lines were examined. RNA was isolated using the GeneMATRIX Universal RNA purification kit (EURx Ltd. Gdansk, Poland) as described by the manufacturer's protocol. Reverse transcription was performed using M-MLV reverse transcriptase (Invitrogen by Thermo Fisher Scientific, Waltham, MA, USA) and a thermal cycler (Veriti 96-well Thermal Cycler) as described in the manufacturer's protocol. Two micrograms of RNA were used for cDNA synthesis. Real-time PCR was performed using the 7900HT Fast Real-Time PCR System (Applied Biosystems, Foster City, CA, USA), Maxima SYBR Green/ROX qPCR Master Mix (Thermo Fisher Scientific,Waltham, MA, USA) and the sequence-specific primers that are indicated in Table 1. Glyceraldehyde-3-phosphate dehydrogenase $(G A D P H), \beta$-actin, hypoxanthine-guanine phosphorribosyltransferase 1 (HRPT1) and beta-2-microglobulin $(\beta 2 M)$ served as the normalizing genes (geometric mean) for the gene expressions being analysed. Gene expressions were analysed using the relative quantification (RQ) method. The RQ method estimates the differences in gene expression against a calibrator (drug-sensitive line) $(\mathrm{RQ}$ of the calibrator $=$ 1). The drug-sensitive $A 2780$ or $W 1$ cell lines were used as the calibrator. The analysis was conducted using the following standard formula: $R Q=2-\Delta \Delta C t$ (where $\Delta \Delta \mathrm{Ct}=\Delta \mathrm{Ct}$ of the sample (drug-resistant line) - $\Delta \mathrm{Ct}$ of the calibrator (drug sensitive line)). The graphs were plotted using Sigma Plot. For amplification, $12.5 \mu \mathrm{L}$ of Maxima SYBR Green/ROX qPCR Master Mix (Thermo Fisher Scientific,Waltham, MA, USA), $1 \mu \mathrm{L}$ of each primer (Oligo, Warsaw, Poland) (Table 1), $9.5 \mu \mathrm{L}$ of water, and $1 \mu \mathrm{L}$ of cDNA solution were mixed together. One RNA sample from each preparation was processed without the RTreaction to provide a negative control in the subsequent PCR reaction. Sample amplification included a hot start $\left(95^{\circ} \mathrm{C}, 15 \mathrm{~min}\right)$ followed by 40 cycles of denaturation at $95^{\circ} \mathrm{C}$ for 15 seconds, annealing at $60^{\circ} \mathrm{C}$ for 30 seconds, and extension at $72^{\circ} \mathrm{C}$ for 30 seconds. After amplification, melt curve analysis was conducted to analyse the product melting temperatures. The amplification products were also resolved using 3\% agarose gel electrophoresis and visualized by ethidium bromide staining.

Table 1. Oligonucleotide sequences used for RQ-PCR analysis.

\begin{tabular}{|c|c|c|c|}
\hline Transcript & Sequence (5'-3' direction) & $\begin{array}{l}\text { ENST number } \\
\text { http://www.ense } \\
\text { mbl.org }\end{array}$ & $\begin{array}{l}\text { Product } \\
\text { size (bp) }\end{array}$ \\
\hline MYOT & $\begin{array}{l}\text { ATCAGGATGCAATCCAGGAG } \\
\text { AGCTGGCAGTCCACTCACTT }\end{array}$ & 00000421631 & $119 \mathrm{bp}$ \\
\hline GADPH & $\begin{array}{l}\text { GAAGGTGAAGGTCGGAGTCA } \\
\text { GACAAGCTTCCCGTTCTCAG }\end{array}$ & 00000229239 & $199 \mathrm{bp}$ \\
\hline$\beta$-actin & $\begin{array}{l}\text { TCTGGCACCACACCTTCTAC } \\
\text { GATAGCACAGCCTGGATAGC }\end{array}$ & 00000331789 & $169 \mathrm{bp}$ \\
\hline HRPT1 & $\begin{array}{l}\text { CTGAGGATTTGGAAAGGGTG } \\
\text { AATCCAGCAGGTCAGCAAAG }\end{array}$ & 00000298556 & $156 \mathrm{bp}$ \\
\hline$\beta 2 \mathrm{M}$ & $\begin{array}{l}\text { CGCTACTCTCTCTTTCTGGC } \\
\text { ATGTCGGATGGATGAAACCC }\end{array}$ & 00000558401 & $133 \mathrm{bp}$ \\
\hline
\end{tabular}

\section{Protein isolation from cell culture and media}

The cells $\left(1 \times 10^{6}\right.$ cells $/ 25 \mu$ L lysis buffer $)$ were lysed in RIPA buffer containing protease inhibitor cocktail (Roche Diagnostics $\mathrm{GmbH}$, Mannheim, Germany) for $60 \mathrm{~min}$ on ice at $4^{\circ} \mathrm{C}$. The lysates were centrifuged at $12000 \times \mathrm{g}$ for $15 \mathrm{~min}$ at $4^{\circ} \mathrm{C}$, and protein concentrations were determined using the Bradford protein assay system (Bio-Rad Laboratories, Hemel Hempstead, UK). To isolate proteins from media, cells were cultured in serum-free media for 72 hours. Next, the media were centrifuged at $15000 \mathrm{rpm}$ for $30 \mathrm{~min}$ at RT. Then, the supernatants were transferred to Amicon Ultra-15 3K centrifuge filter devices and centrifuged according to the manufacturer's instructtions (60 min, $4000 \times \mathrm{g}$, RT, swinging-bucket rotor).

\section{SDS-PAGE and Western Blot Analysis of MYOT, COL1A2 and COL15A1}

Thirty micrograms of protein from each sample was resuspended in $4 \times$ loading buffer (Bio-Rad Laboratories, Hemel Hempstead, UK) and incubated at RT for $20 \mathrm{~min}$. The resuspended protein was loaded into each well and separated on a $4-20 \%$ mini-PROTEAN ${ }^{\circledR} \mathrm{TGX}^{\mathrm{TM}}$ precast gel using the 
SDS-PAGE technique. The proteins were transferred to a nitrocellulose membrane, blocked with $5 \%$ milk in TBS/Tween (0.1 M Tris-HCl, $0.15 \mathrm{M} \mathrm{NaCl}, 0.1 \%$ Tween 20) and immunodetected using mouse anti-MYOT Ab at 1:1000 dilution, goat anti-COL1A2 $\mathrm{Ab}$ at 1:1000 dilution or goat anti-COL15A1 at 1:1000 dilution and the appropriate HRP-conjugated secondary $\mathrm{Ab}$. Chemiluminescence detection of the separated bands was performed using an enhanced chemical luminescence (ECL) kit (Femto Super Signal Reagent) and Hyperfilm ECL (GE Healthcare, Buckinghamshire, UK). To normalize protein loading in the lanes, the membranes were stripped and reblotted with rabbit anti-GADPH Ab, from Santa Cruz Biotechnology, at a 1:1000 dilution and goat anti-rabbit $\mathrm{HRP}$-conjugated $\mathrm{Ab}$.

\section{Immunofluorescence analysis}

The cells were cultured on microscopic glass slides and grown to a near-confluent state. Afterwards, the cells were fixed in $4 \%$ PFA in PBS for $10 \mathrm{~min}$ at room temperature, permeabilized in ice-cold acetone/methanol (1:1) for $10 \mathrm{~min}$ at $-20^{\circ} \mathrm{C}$, rinsed with PBS and blocked in 3\% BSA for $45 \mathrm{~min}$. Anti-MYOT primary antibody (mouse monoclonal anti-MYOT antibody, 1:200 Santa Cruz Biotechnology, Santa Cruz, USA) was used for detection along with the corresponding green dye-labelled secondary antibody (MFP488, donkey anti-goat IgG, 1:200, MoBiTec, Goettingen, Germany). Afterwards, the cells were washed three times with PBS and sealed with DAPI-containing mounting medium (Santa Cruz Biotechnology, Santa Cruz, CA, USA). The expression of MYOT was analysed under a fluorescence microscope (Zeiss Axio-Imager.Z1) by pseudo-colour representations of fluorescence intensity for DAPI at $365 \mathrm{~nm}$ excitation and $420 \mathrm{~nm}$ emission wavelengths (blue) and for MFP488 at $470 \mathrm{~nm}$ excitation and 525 $\mathrm{nm}$ emission wavelengths (green).
A

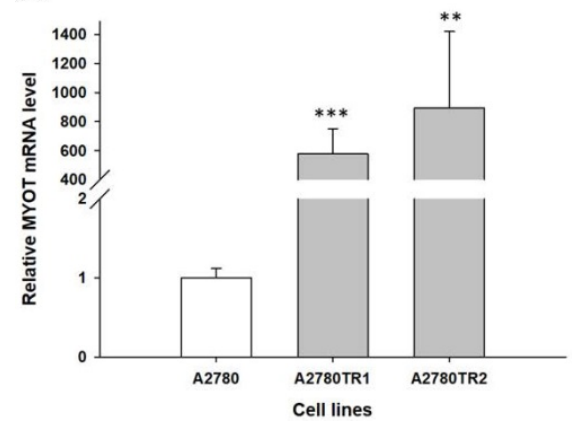

B

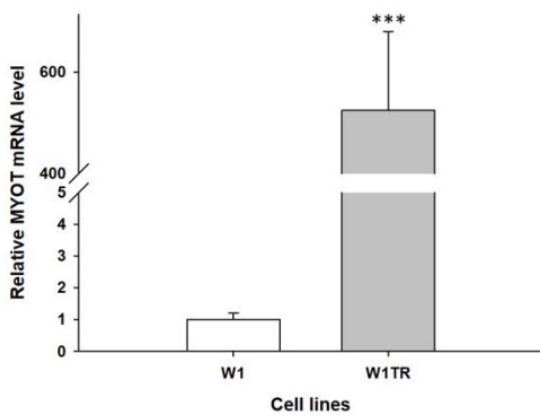

Figure 1. Expression analysis (Q-PCR) of the MYOT gene in the A2780 (A) and W1 (B) TOP-resistant cell sublines. The figure presents the relative gene expression in the resistant cell lines (grey bars) with respect to that in the sensitive cell line (white bars), which was assigned a value of 1 . The values were considered significant at $* * p<0.01$ and $* * * p<0.001$.

\section{Statistical analysis}

Statistical analysis was performed using Microsoft Excel software. The statistical significance of the differences was determined using the Student's t-test, and p-values of 0.05 or less were considered to be statistically significant.

\section{Results}

\section{Analyses of MYOT gene expression in TOP-resistant ovarian cancer cell lines}

To determine whether the development of TOP-resistance is associated with the MYOT overexpression, the expression of the MYOT mRNA was determined in TOP-resistant sublines. We observed high and statistically significant increase of the MYOT transcript in both A2780 TOP-resistant sublines $(p<0.001$ in A2780TR1 and $p<0.001$ in A2780TR2) (Figure 1A) and in W1 TOP-resistant subline $(p<0.001)$ (Figure 1B).

\section{Immunofluorescence of the MYOT protein expressed in resistant cell lines}

To confirm the presence of the MYOT protein in the investigated cell lines, we performed fluorescence analysis of its expression in A2780, W1 and drug-resistant cell lines. A low fluorescence signal was present in the $\mathrm{A} 2780$ and $\mathrm{W} 1$ cell lines. In the A2780TR1, A2780TR2 and W1TR cell lines, we observed increase in fluorescence intensity (Figure 2).

\section{Western blot analysis of MYOT}

The elevated expression of MYOT at the protein level was also confirmed by Western blot analysis. In cell lysates we observed clear increase in MYOT bounds intensity in all TOP-resistant cell lines. Furthermore, a clear correlation between transcript and protein level was observed (Figure 3A). Next, we analyzed the presence of MYOT protein in the cell culture medium. Clear increase of bound intensity was observed in A2780TR1 and A2780TR2 cell line in comparison to control - A2780 cell line. Here we also observed a correlation between transcript and secreted protein level. Stronger MYOT bound was also observed in W1TR cell line than in W1 TOP-sensitive cell line (Figure 3B). The amount of secreted MYOT protein was much higher in W1 and W1TR cell lines than in A2780 and TOP-resistant sublines. 


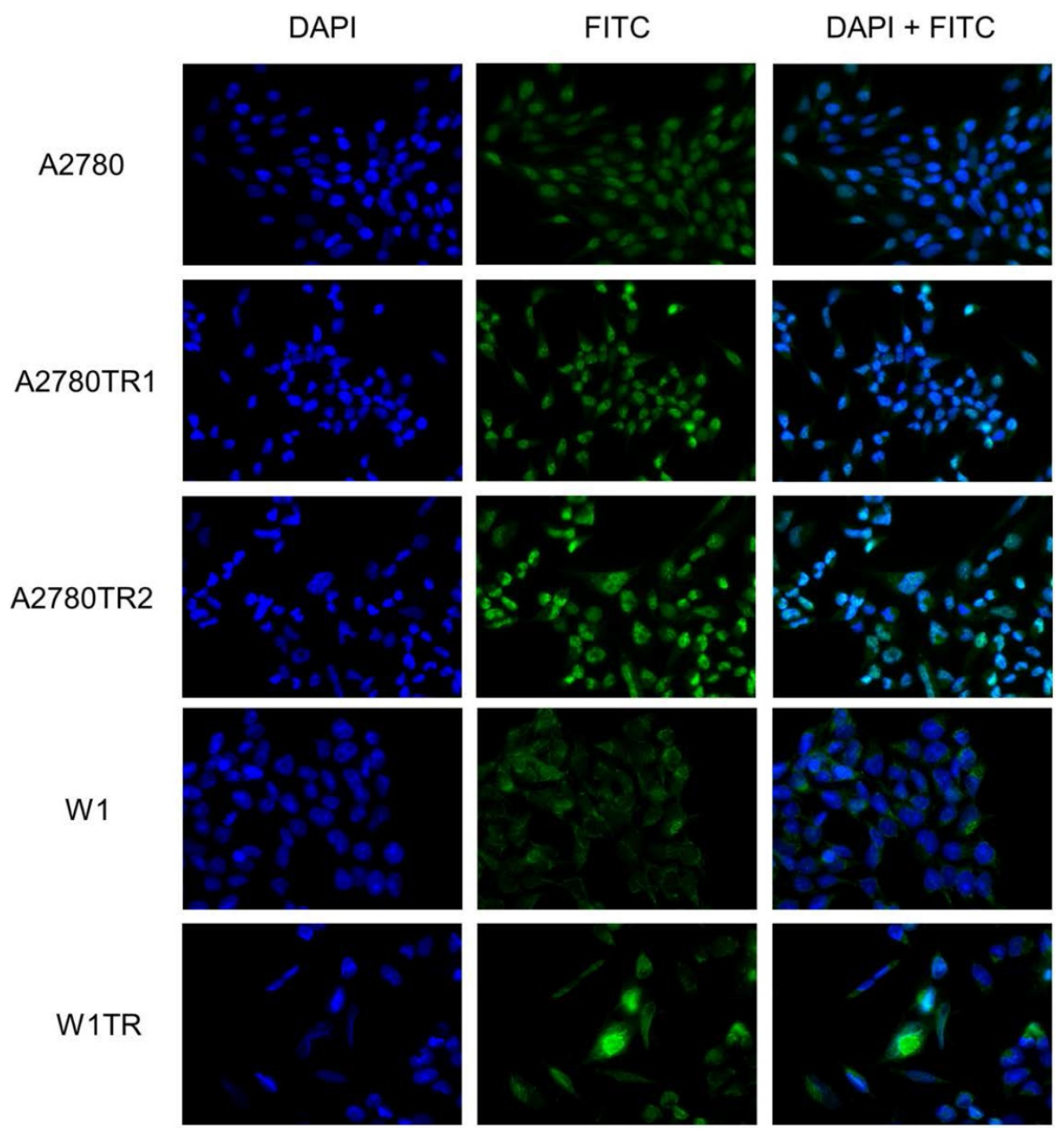

Figure 2. Immunofluorescence visualization of MYOT expression in the A2780, A2780TR1, A2780TR2, W1 and WITR cell lines. MYOT was detected using the anti-MYOT antibody and MFP488 secondary antibody (green). To visualize the cell nuclei, the cells were mounted with a DAPI-containing mounting medium (blue).

Western blot analysis of secreted COL1A2 and COLI5AI

Previously we observed increased expression of COL1A2 in both A2780 TOP-resistant cell lines and increased expression of COL15A1 in A2780TR2 and W1TR cell lines [27]. In this study we wanted to check whether both types of collagen are secreted to the cell culture media. Western blot analysis showed clear increase of COL1A2 bound intensity in A2780TR1 and A2780TR2 cell lines (Figure 4A). Increased COL15A1 bound intensity was observed in A2780TR2 and W1TR cell lines in comparison to control (Figure 4B). Secreted collagens were also present in drug sensitive cell lines although at lower level (Figure 4 A, B).

\section{Discussion}

The most significant problem with treatment efficacy of cancer is resistance of cancers to chemotherapeutic agents. Some cancers are intrinsically resistance to chemotherapy, however most develop drug resistance during treatment. Most of the researchers focus only on cellular mechanism of drug resistance like expression of drug transporters from $A B C$ family and these mechanisms are abundantly described in the literature [36]. However other mechanisms that are related to expression of ECM molecules and tissue specific mechanisms of drug resistance seem to be even more important from the clinical point of view. Expression of ECM molecules can adopt drug resistance in at least three ways. Dense structure of molecules in ECM can block drug diffusion [12], some drugs can directly bind to ECM molecules that limit their availability to tumor cells [13], and some ECM molecules like collagens can bind to integrins and induce CAM-DR [14]. In physiological condition ECM components are produced mainly by cells of connective tissue. 
However, in pathological condition e.g. cancer, expression of ECM components was also observed right in cancer cells in vivo [14, 37] as well as in ovarian cancer and breast cancer cell lines [17-19]. Recently we also observed that ECM components like COL3A1 and lumican (LUM) [26] are secreted to cell culture medium in drug resistance ovarian cancer cell line. In a state where cancer cells of epithelial origin express molecules typical for connective tissue it can be assumed that these cells are able to express genes characteristic for other types of tissues. In pathological state these molecules can play a different role from that observed in physiological condition. The incomparable tool for looking for the new genes related to drug resistance is the RNA microarray. Recently, with the use of RNA microarray, we identified a set of new genes with expression characteristic to resistance to drugs used in ovarian cancer chemotherapy [35]. Among them we identified a MYOT with increased expression in all three TOP-resistant cell lines.

TOP is one of the chemotherapeutic agents used in second line of ovarian cancer therapy in case of platinum and taxane resistant patients [3,5]. The well described mechanisms of TOP-resistance are those related to decreased expression of DNA topoisomerase I and upregulation BCRP protein encoded by the $A B C G 2$ gene $[8,9,38]$. However, the new genes related to drug resistance are permanently described in literature. Recently we also described expression of a few new genes in TOP-resistant cell lines [28, 29]. Here we describe detailed expression of MYOT at mRNA and protein level.

We observed very high (from 500 to 800 -fold)

A

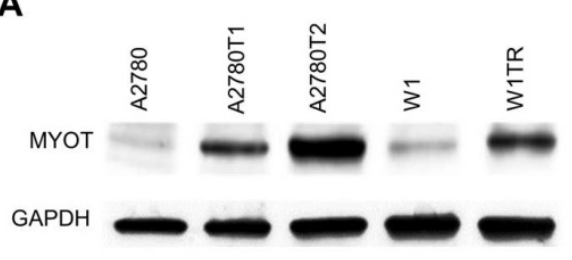

Figure 3. MYOT protein expression analysis in the $A 2780, \mathrm{~W} 1$ and drug-resistant cell lines $(\mathrm{A})$ and their corresponding media (B). The cellular proteins and proteins isolated from the media were separated using 7\% PAGE and transferred to a PVDF membrane, which was then immunoblotted with either primary Ab or HRP-conjugated secondary Ab. A primary anti-GADPH Ab was used as a loading control for the cell lysates.

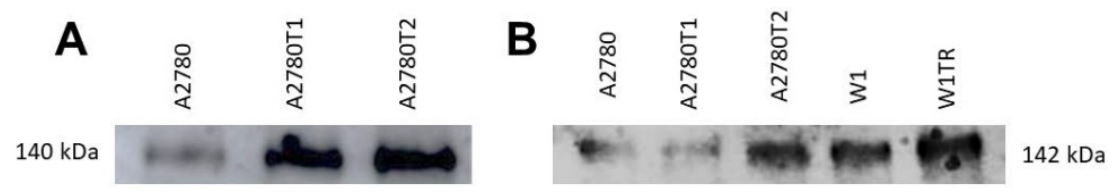

Figure 4. COLIA2 (A) and COL15A1 (B) protein expression analysis in cell culture media. The proteins isolated from the media were separated using 7\% PAGE and transferred to a PVDF membrane, which was then immunoblotted with either primary $A b$ or HRP-conjugated secondary $A b$. increase in MYOT mRNA level in three investigated TOP-resistant ovarian cancer cell lines. Immunofluorescence analysis confirmed elevated expression of MYOT protein in those cell lines, which indicates that MYOT is equally expressed in all cells. Being aware that immunofluorescence is not a quantitative method in next step we performed western blot experiments to compare the protein expression levels of MYOT between investigated cell lines. In cell lysates western blot results showed clear increase in MYOT protein expression and high correlation with mRNA level suggesting that expression of MYOT is regulated at mRNA level. Because in our previous study we detected COL3A1 and LUM secreted into the cell culture medium [26] we were interested whether MYOT can be also secreted or not. Therefore, we proceed with western blot analysis performed for cell culture medium and indeed, we confirmed MYOT protein presence in cell culture media. Clear correlation between protein level in cell lysates and medium was observed in A2780 and TOP-resistant sublines. For W1TR cell culture medium we also observed increase in MYOT protein level in comparison to control However, the elevated MYOT protein level seems to be much higher in W1 cell culture medium than in A2780 cell culture medium. MYOT has been described as a structural protein in skeletal and cardiac muscle [30] and to our knowledge not as a secreted protein. Therefore, our next step was to determine whether other secreted protein could be detectable in corresponding cell culture medium. Previously we described expression of several collagens in our drug resistance cell lines [27]. Among TOP-resistant cell lines we observed increased expression of COL1A2 in A2780TR1 and A2780TR2 cell lines as well as increased expression of COL15A1 in A2780TR2 and W1TR cell lines at mRNA and protein level [27]. Those results led us to investigate collagens as secreted proteins in cell culture media in the current study. With a reference to previous results we could observe clear increase in COL1A2 protein in cell culture media from A2780TR1 and A2780TR2 cell lines and increased level of secreted COL15A1 in cell culture medium from A2780TR2 and W1TR cell lines. Previously we also described presence of extracellular COL3A1 in W1TR cell line [26, 27]. To our knowledge, this is the first report about MYOT expression in drug resistant cell lines and 
moreover about MYOT as a secretory protein found in the cancer cell culture media. We undertook a literature search very carefully and we did not find any information about expression of MYOT in any cancer or about MYOT as a secretory protein. Therefore, we could not compare or refer our results to other research and this result need further validation.

Taken together, cells do not use superfluous energy for expression of proteins that are not required for their survival. Increasing evidence indicates that elevated levels of MYOT mRNA and protein expression could be associated with the ability to resist TOP treatment by cancer cells. This finding is consistent with our previous study where we described expression of SAMD4 gene in all investigated TOP-resistant cell lines [28]. This gene encodes regulatory protein responsible for repression of translation and transcript decay by binding to SRE elements on target transcripts [39]. During development of Drosophila embryo it induces a degradation of two-thirds target maternal mRNAs [40]. It is possible that in stress condition, like treatment with cytotoxic drugs, SAMD4 targets less important transcripts leading to inhibition of their translation. As a result, the cell can focus all attention on expression of genes responsible for drug resistance, even those that are normally not expressed in epithelial cells- like MYOT.

Since we could observe that MYOT as well as COL1A2 and COL15A1 are secreted to cell culture medium the issue of the role of these proteins in drug resistance has been raised. Three different possibilities were taken under consideration. The first possibility assumes the role of intracellular and extracellular MYOT and collagens in binding TOP molecules and therefore in limiting its availability for cancer cells' targets. It is highly probable especially in case of MYOT since its expression was demonstrated in all three TOP-resistant cell lines. That remains consistent with other reports where binding of PAC, DOX and VIN to extracellular molecules resulted in their limited availability to tumor cells [12]. Therefore, the potential role of MYOT in cytotoxic drug binding needs further investigation. The second possibility to be considered is a limited drug diffusion. In a previous study, elevated expression of some ECM components, especially collagens were found to limit drug diffusion in cancer tissue [11, 12 41, 42]. Therefore, it is possible that presence of MYOT, COL1A2 and COL15A1 limit TOP diffusion among cancer cells examined in present study. However, to prove this hypothesis further analyses with the use of more advanced models of study, like cells growing in 3D cell culture condition, should be applied [43]. Finally, the third assumption concerns the role of extracellular MYOT and collagens in CAM-DR [14, 37]. Such mechanism of drug resistance has been observed both in in vivo and in vitro condition. Cancer cells utilize a surface receptors - like integrins - and interact with their microenvironment that results in inhibition of drug induced apoptosis. It has been observed that interaction of $\beta 1$-integrin with ECM leads to resistance of cancer to melphalan and DOX [15]. Moreover, it was demonstrated that interaction of cancer cells with ECM components induces drug resistance in vitro as well. The culture of drug sensitive A2780 ovarian cancer cell line on COL6A3 coated dishes induced resistance to CIS [16]. This mechanism is not restricted only to collagens but was also observed for pancreatic cancer cells growing on the surface composed of fibronectin and laminin, where cancer cells became resistant to DOX, CIS and 5-fluorouracil (5-FU) [44] and for breast cancer cell line MDA-MB-231 growing on the surface of fibronectin and type I collagen that became resistant to PAC [45]. MYOT is a structural protein that interacts with many other proteins in cells [46]. IgG-like domains of MYOT are responsible for dimerization and binding of other proteins like F- and G-actin [31, 47] or filamin C [32]. On the other hand, N-terminal sequence of MYOT is responsible for binding calmodulin-like domain in a-actin-2 [48]. Taking all these findings together it can be assumed that MYOT has a potential to interact with other cellular molecules including cell surface receptors and in this way, can induce CAM-DR. This however requires further studies.

It will be of great interest to elucidate the role of MYOT in TOP-resistance of cancer cells. Therefore, as a continuation of current research a knock-down and overexpression experiments with the use of animal model will be made. In 2D cell culture conditions cancer cells prefer cell- to tissue-specific mechanism of drug resistance. In vivo model allows cancer cells to form tumor-like structure with more significant role of ECM components in drug resistance development. A more thorough understanding of the role of MYOT as a novel agent in TOP-resistance may have an influence on the research leading to more effective treatment of ovarian cancer.

\section{Conclusions}

In conclusion, the research has found elevated levels of MYOT expression in response to TOP treatment in ovarian cancer cell lines. Increased expression of MYOT in all TOP-resistant cell lines suggests its specific role in acquiring resistance to TOP by cancer cells. To our knowledge, this is also the first report about MYOT described as a secretory protein found in the cancer cell culture media. The 
significance of MYOT in TOP or other drug resistance was not described previously and requires further investigation.

\section{Abbreviations}

TOP: Topotecan; MYOT: Myotilin; EOC: Epithelial Ovarian Cancer; ECM: Extracellular Matrix; CAM: Cell Adhesion Molecule; TR: Topotecan Resistance.

\section{Acknowledgements}

This study was supported by Grant No. 2016/22/E/NZ5/00381 from the National Science Centre, Kraków, Poland.

\section{Author Contributions}

KS and AK prepared all cell lines and cell culture analyses and were the main contributors to the writing of the manuscript; KW was involved western blot experiments; MŚ was involved in Q-PCR experiments; $\mathrm{MN}$ was involved in the data interpretation and revision of the manuscript; JB was involved in the data interpretation and revision of the manuscript; RJ was involved in the data interpretation and revision of the manuscript.

\section{Competing Interests}

The authors have declared that no competing interest exists.

\section{References}

1. Webb PM, Jordan SJ. Epidemiology of epithelial ovarian cancer. Best Pract Res Clin Obstet Gynaecol. 2017; 41: 3-14

2. Hennessy BT, Coleman RL, Markman M. Ovarian cancer. Lancet. 2009; 374: 1371-1382.

3. Webber K, Friedlander M. Chemotherapy for epithelial ovarian, fallopian tube and primary peritoneal cancer. Best Pract Res Clin Obstet Gynaecol. 2016; 41: 126-138.

4. Parmar MK, Ledermann JA, Colombo N, du Bois A, Delaloye JF, Kristensen GB, et al. Paclitaxel plus platinum-based chemotherapy versus conventional platinum-based chemotherapy in women with relapsed ovarian cancer: the ICON4/AGO-OVAR-2.2 trial. Lancet. 2003; 361: 2099-2106.

5. Sehouli J, Stengel D, Oskay-Oezcelik G, Zeimet AG, Sommer H, Klare P, et al. Nonplatinum topotecan combinations versus topotecan alone for recurrent ovarian cancer: results of a phase III study of the North-Eastern German Society of Gynecological Oncology Ovarian Cancer Study Group. J Clin Oncol. 2008; 26: 3176-3182.

6. Ferrandina G, Ludovisi M, Lorusso D, Pignata S, Breda E, Savarese A, et al. Phase III trial of gemcitabine compared with pegylated liposomal doxorubicin in progressive or recurrent ovarian cancer. J Clin Oncol. 2008; 26: 890-896.

7. Choi $\mathrm{CH}$. ABC transporters as multidrug resistance mechanisms and the development of chemosensitizers for their reversal. Cancer Cell Int. $2005 ; 5$ : e30.

8. Januchowski R, Sterzyńska K, Zaorska K, Sosińska P, Klejewski A, Brązert M, et al. Analysis of MDR genes expression and cross-resistance in eight drug resistant ovarian cancer cell lines. J Ovarian Res. 2016; 9: e65.

9. Januchowski R, Wojtowicz K, Sujka-Kordowska P, Andrzejewska M, Zabel M. MDR gene expression analysis of six drug-resistant ovarian cancer cell lines. Biomed Res Int. 2013; e241763.

10. Tannock IF, Lee CM, Tunggal JK, Cowan DS, Egorin MJ. Limited penetration of anticancer drugs through tumor tissue: a potential cause of resistance of solid tumors to chemotherapy. Clin Cancer Res. 2002; 8: 878-884.

11. Chauhan VP, Stylianopoulos T, Boucher Y, Jain RK. Delivery of molecular and nanoscale medicine to tumors: transport barriers and strategies. Annu Rev Chem Biomol Eng. 2011; 2: 281-298.

12. Di Paolo A, Bocci G. Drug distribution in tumors: mechanisms, role in drug resistance, and methods for modication. Curr Oncol Rep. 2007; 9: 109-114.

13. St Croix B, Kerbel RS. Cell adhesion and drug resistance in cancer. Curr Opin Oncol. 1997; 9: 549-556.
14. Correia AL, Bissell MJ. The tumor microenvironment is a dominant force in multidrug resistance. Drug Resist Updat. 2012; 15: 39-49.

15. Sethi T, Rintoul RC, Moore SM, MacKinnon AC, Salter D, Choo C, et al. Extracellular matrix proteins protect small cell lung cancer cells against apoptosis: a mechanism for small cell lung cancer growth and drug resistance in vivo. Nat Med. 1999; 5: 662-668.

16. Sherman-Baust CA, Weeraratna AT, Rangel LB, Pizer ES, Cho KR, Schwartz $\mathrm{DR}$, et al. Remodeling of the extracellular matrix through overexpression of collagen VI contributes to cisplatin resistance in ovarian cancer cells. Cancer Cell. 2003; 3: 377-386.

17. Januchowski R, Zawierucha P, Ruciński M, Zabel M. Microarray-based detection and expression analysis of extracellular matrix proteins in drug-resistant ovarian cancer cell lines. Oncol Rep. 2014; 32: 1981-1990.

18. Januchowski R, Zawierucha P, Ruciński M, Nowicki M, Zabel M. Extracellular matrix proteins expression profiling in chemoresistant variants of the A2780 ovarian cancer cell line. Biomed Res Int. 2014; e365867.

19. Iseri OD, Kars MD, Arpaci F, Gündüz U. Gene expression analysis of drug-resistant MCF-7 cells: implications for relation to extracellular matrix proteins. Cancer Chemother Pharmacol. 2009; 65: 447-455.

20. Rosen VM, Guerra I, McCormack M, Nogueira-Rodrigues A, Sasse A, Munk VC, et al. Systematic Review and Network Meta-Analysis of Bevacizumab Plus First-LineTopotecan-Paclitaxel or Cisplatin-Paclitaxel Versus Non-Bevacizumab-Containing Therapies in Persistent, Recurrent, or Metastatic Cervical Cancer. Int J Gynecol Cancer. 2017; 27: 1237-1246.

21. Tas F, Derin D, Guney N, Camlica H, Aydiner A, Topuz E. Addition of topotecan to standard cisplatin/etoposide combination in patients with extended stage small cell lung carcinoma. Lung Cancer. 2007; 57: 79-83.

22. Pommier $\mathrm{Y}$, Leo $\mathrm{E}$, Zhang $\mathrm{H}$, Marchand $\mathrm{C}$. DNA topoisomerases and their poisoning by anticancer and antibacterial drugs. Chem Biol. 2010; 17: 421-433.

23. Pommier Y, Sun Y, Huang SN, Nitiss JL. Roles of eukaryotic topoisomerases in transcription, replication and genomic stability. Nat Rev Mol Cell Biol. 2016; 17: 703-721.

24. Staker BL, Hjerrild K, Feese MD, Behnke CA, Burgin ABJr, Stewart L. The mechanism of topoisomerase I poisoning by a camptothecin analog. Proc Natl Acad Sci USA. 2002; 99: 15387-15392.

25. Rubin EH, Li T, Duan P, Liu LF. Cellular resistance to topoisomerase poisons. Cancer Treat Res. 1996: 243-260.

26. Klejewski A, Sterzyńska K, Wojtowicz K, Świerczewska M, Partyka M, Brązert $\mathrm{M}$, et al. The significance of lumican expression in ovarian cancer drug-resistant cell lines. Oncotarget. 2017; 8: 74466-74478.

27. Januchowski R, Świerczewska M, Sterzyńska K, Wojtowicz K, Nowicki M, Zabel M. Increased expression of several collagen genes is associated with drug resistance in ovarian cancer cell lines. J Cancer. 2016; 25: 1295-1310.

28. Klejewski A, Świerczewska M, Zaorska K, Brązert M, Nowicki M, Zabel M, et al. New and Old Genes Associated with Topotecan Resistance Development in Ovarian Cancer Cell Lines. Anticancer Res. 2017; 37: 1625-1636.

29. Świerczewska M, Klejewski A, Wojtowicz K, Brązert M, Iżycki D, Nowicki M, et al. New and Old Genes Associated with Primary and Established Responses to Cisplatin and Topotecan Treatment in Ovarian Cancer Cell Lines. Molecules. 2017; 22: e1717.

30. Salmikangas P, Mykkanen OM, Gronholm M, Heiska L, Kere J, Carpen O. Myotilin, a novel sarcomeric protein with two Ig-like domains, is encoded by a candidate gene for limb-girdle muscular dystrophy. Hum. Mol. Genet. 1999; 8: 1329-1336.

31. Von Nandelstadh P, Grönholm M, Moza M, Lamber A, Savilahti H, Carpén O. Actin-organising properties of the muscular dystrophy protein myotilin. Exp Cell Res. 2005; 310: 131-139.

32. Van Der Ven PF, Wiesner $S$, Salmikangas $P$, Auerbach D, Himmel M, Kempa $\mathrm{S}$, et al. Indications for a novel muscular dystrophy pathway. $\gamma$-filamin, the muscle-specific filamin isoform, interacts with myotilin. J. Cell Biol. 2000; 151: 235-248.

33. Gontier $\mathrm{Y}$, Taivainen A, Fontao L, Sonnenberg A, Van Der Flier A, Carpen O, et al. The Z-disc proteins myotilin and FATZ-1 interact with each other and are connected to the sarcolemma via muscle-specific filamins. J. Cell Sci. 2005; 118: 3739-3749.

34. Selcen D. Myofibrillar myopathies. Neuromuscul Disord. 2011; 21: 161-171.

35. Januchowski R, Sterzyńska K, Zawierucha P, Ruciński M, Świerczewska M, Partyka M, et al. Microarray-based detection and expression analysis of new genes associated with drug resistance in ovarian cancer cell lines. Oncotarget. 2017; 8: 49944-49958.

36. Stavrovskaya AA. Cellular mechanism of multidrug resistance of tumor cells. Biochemistry. 2000; 65: 95-106.

37. Morin PJ. Drug resistance and the microenvironment: nature and nurture. Drug Resist Updat. 2003; 6: 169-172.

38. Robey RW, Polgar O, Deeken J, To KW, Bates SE. ABCG2: determining its relevance in clinical drug resistance. Cancer Metastasis Rev. 2007; 26: 39-57.

39. Pinder BD, Smibert CA. Smaug: an unexpected journey into the mechanisms of post-transcriptional regulation. Fly (Austin). 2013; 7: 142-145.

40. Tadros W, Goldman AL, Babak T, Menzies F, Vardy L, Orr-Weaver T, et al. SMAUG is a major regulator of maternal mRNA destabilization in Drosophila and its translation is activated by the PAN GU kinase. Dev Cell. 2007; 12: 143-155.

41. Netti PA, Berk DA, Swartz MA, Grodzinsky AJ, Jain RK. Role of extracellular matrix assembly in interstitial transport in solid tumors. Cancer Res. 2000; 60: 2497-2503. 
42. Stylianopoulos T, Diop-Frimpong B, Munn LL, Jain RK. Diffusion anisotropy in collagen gels and tumors: the effect of fiber network orientation. Biophys J. 2010; 99: 3119-3128.

43. Tung YC, Hsiao AY, Allen SG, Torisawa YS, Ho M, Takayama S. High-throughput 3D spheroid culture and drug testing using a 384 hanging drop array. Analyst. 2011; 136: 473-478.

44. Miyamoto $H$, Murakami $T$, Tsuchida $K$, Sugino $H$, Miyake $H$, Tashiro S. Tumor-stroma interaction of human pancreatic cancer: acquired resistance to anticancer drugs and proliferation regulation is dependent on extracellular matrix proteins. Pancreas. 2004; 28: 38-44.

45. Aoudjit F, Vuori K. Integrin signaling inhibits paclitaxel-induced apoptosis in breast cancer cells. Oncogene. 2001; 20: 4995-5004.

46. Puž V, Pavšič M, Lenarčič B, Djinović-Carugo K. Conformational plasticity and evolutionary analysis of the myotilin tandem Ig domains. Sci Rep. 2017; 7: e3993.

47. Salmikangas $\mathrm{P}$, van der Ven PF, Lalowski M, Taivainen A, Zhao F, Suila H, et al. Myotilin, the limb-girdle muscular dystrophy 1A (LGMD1A) protein, cross-links actin filaments and controls sarcomere assembly. Hum Mol Genet. 2003; 12: 189-203.

48. Beck MR, Otey CA, Campbell SL. Structural Characterization of the Interactions between Palladin and a-Actinin. J Mol Biol. 2011; 413: 712-725. 